\title{
Interaction of energetic particles and dust grains with asteroidal surfaces
}

\author{
J. N. Goswami \\ Physical Research Laboratory, Ahmedabad - 380 009, India \\ (Received March 20, 2001; Revised September 30, 2001; Accepted October 1, 2001)
}

\begin{abstract}
Low energy solar wind (SW) and solar energetic particles (SEP) interact with material exposed on the surface of planetary objects devoid of an atmosphere and a global magnetic field such as the Moon and the asteroids. Micrometeoroid impacts will produce microcraters on samples exposed on the surfaces of these bodies and also cause microscale erosion and at times catastrophic rupture of the target rocks and soil grains. The impacting flux of meteoroids thus controls the effective SW and SEP exposure durations of samples on the lunar and asteroidal surfaces. The lunar samples contain abundant records of energetic particle interactions and micrometeoroid impacts while such records found in gas-rich meteorites can be considered to be representative of asteroidal samples. These records allow us to infer the composition and intensity of SW and SEP in the past as well as surface dynamical processes operating on the Moon and asteroids at different epochs. However, unlike the lunar records that span the time interval from the present to several billion years in the past, the asteroidal (gas-rich meteorite) records provide information only for some distant epochs in the past. We do not have much information about the contemporary energetic particle and dust grain environment in the asteroidal region. The Muses- $\mathrm{C}$ mission, that will bring back near surface samples from an asteroid, will be extremely important in this regard.
\end{abstract}

\section{Introduction}

Direct exploration and study of energetic particles and dust grains present in the interplanetary space using earth orbiting as well as interplanetary spacecrafts have become feasible during the last three decades with the advent of the space age. However, to obtain information about their nature and characteristics at more distant epochs, we have to rely on indirect approaches. Our present understanding of the past intensity and composition of energetic particles present in the interplanetary space is mainly based on studies of interaction records of these particles with lunar samples and meteorites (see, e.g., Lal, 1972; Reedy et al., 1983; Goswami et al., 1984; Caffee et al., 1988, 1991; Goswami, 1991). Similarly, studies of micrometeoroid impact induced records in lunar samples and meteorites also provide useful information about the nature of the dust grains present in the interplanetary space at different epochs in the past (see, e.g., Gault et al., 1972, 1974; Brownlee and Rajan, 1973; Morrison and Zinner, 1976, 1977; Goswami et al., 1976, 1984; Ashworth, 1978; McDonnell, 1978; Grun et al., 1985). The fossil records of energetic particle interactions and of micrometeoroid impacts preserved in the lunar samples and meteorites also allow us to infer about the surface dynamical processes in the parent bodies of these samples, i.e., the Moon and the asteroids. In this paper a brief review of the current status of our knowledge in this field is presented along with a discussion on what more we may be able to learn from studies of samples from asteroidal surface that will be retrieved by the Muses-C mission.

Copy right (C) The Society of Geomagnetism and Earth, Planetary and Space Sciences (SGEPSS); The Seismological Society of Japan; The Volcanological Society of Japan; The Geodetic Society of Japan; The Japanese Society for Planetary Sciences.

\section{Energetic Particles and Dust grains in Inter- planetary Space}

Energetic particles present in the interplanetary space comprise of two main components; the solar component and the extra-solar (galactic, interstellar) component. The Sun continuously emits low-energy (KeV/amu) ions in the form of solar-wind (SW), and sporadically releases higher energy (MeV/amu) ions termed as solar energetic particles (SEP) during solar flare events. The extra-solar component of energetic particles consists of the much higher energy ( $\sim$ a few GeV/amu) galactic cosmic rays (GCR), and a lowenergy ( $<30 \mathrm{MeV} / \mathrm{amu})$, low intensity component, known as the anomalous cosmic rays (ACR). The compositions of the SW, SEP and GCR are very close to solar composition, while the ACR particles have very unusual composition and are enriched in elements of high ionization potential such as $\mathrm{He}, \mathrm{N}, \mathrm{O}$ and $\mathrm{Ne}$ (see, e.g., Biswas et al., 1993; Klecker et al., 1998). In addition to these well-defined components, presence of energetic particle accelerated by interplanetary shocks, solar wind pick-up ions and ambient thermal and supra-thermal particles of solar origin have also been detected in the interplanetary space. We shall restrict our discussion primarily to the interactions of SW and SEP components with planetary materials as these components dominate the time-averaged fluxes of energetic particles in interplanetary space at extremely low and intermediate energies (Fig. 1).

Cometary debris is considered to be the primary source of interplanetary dust particles. Additional contributions come from collisional break-up of small bodies within the asteroidal belt as well as from dust of interstellar origin that are characterized by high orbital velocities due to their hyperbolic trajectories. The physical and chemical nature of 


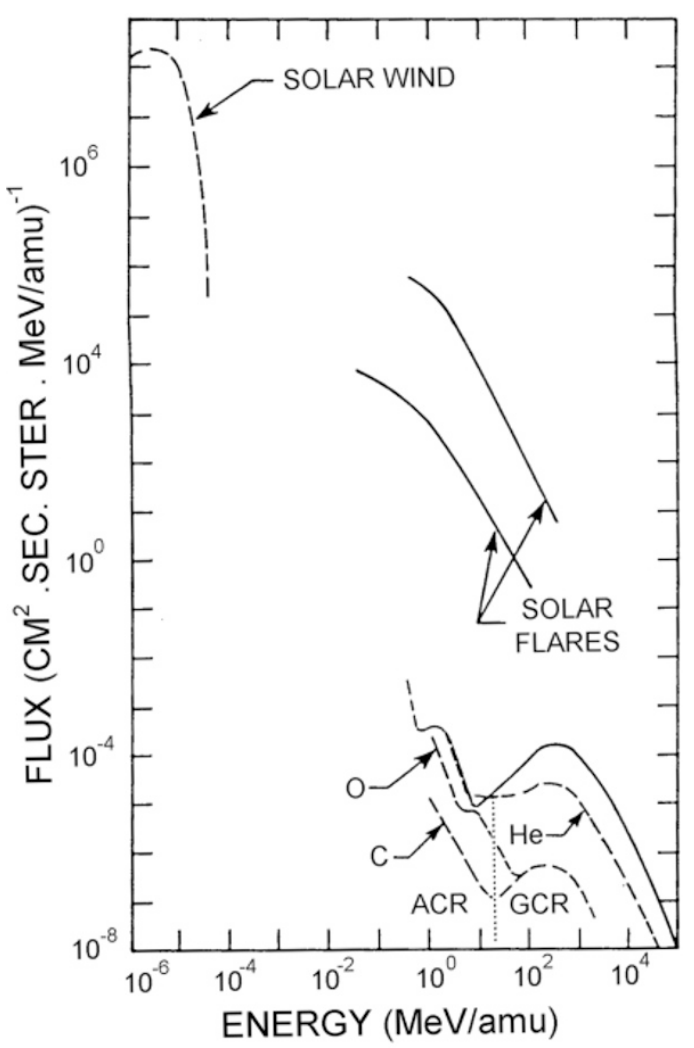

Fig. 1. Contemporary energy spectra of solar wind, SEP, GCR and solar quiet time protons in $1 \mathrm{AU}$ space plotted in the rest frame of the solar wind. The energy spectra of ACR and GCR He, O, and C are also shown. dust particles from asteroidal, cometary and interstellar origin are expected to be different. Concerted effort to sample interplanetary dust grains present in the stratosphere was initiated by NASA a few decades back and the "Stardust" mission is expected to bring back cometary and interstellar dust grain by 2006 . Laboratory studies of interplanetary dust particles suggest that even though their chemical characteristics are similar to some primitive meteorites, their physical characteristics, particularly their porous and fragile nature, are more compatible with a cometary origin (see, e.g., Brownlee, 1985). Some useful information on the nature of the interplanetary dust grains has also come from studies of zodiacal lights and meteors (see, e.g., Weinberg and Sparrow, 1978; Hughes, 1978). Additional information come from studies of impact records of dust grains monitored on board interplanetary spacecrafts and also from laboratory studies of impact records in spacecraft components following their return after long duration exposure in space. However, to infer the properties of interplanetary dust grains at more distant epochs, we have to rely exclusively on the impact records left by them on material exposed on surfaces of planetary bodies like the Moon and asteroids that are devoid of an atmosphere. The long-term averaged flux of interplanetary dust grains inferred from data obtained by using various approaches is shown in Fig. 2.

\section{Interactions of Energetic Particles and Dust Grains with Planetary Material}

The nature of interactions of energetic particles with plan-

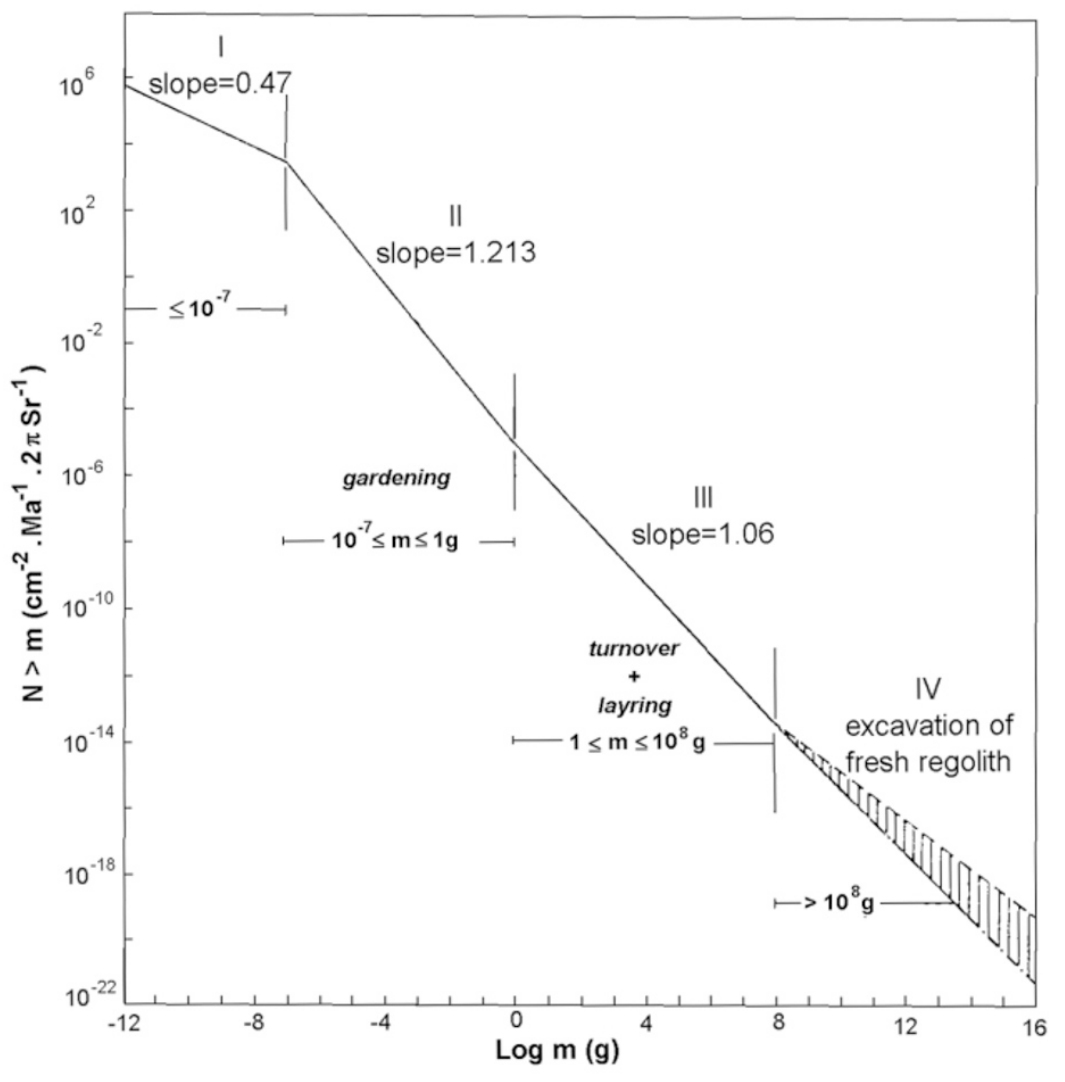

Fig. 2. Cumulative flux of meteoroids in the mass range $10^{-12} \mathrm{~g}$ to $>10^{12} \mathrm{~g}$ in $1 \mathrm{AU}$ space. The approximate ranges of meteoroid masses responsible for gardening, mixing, turnover and blanketing of the lunar regolith are indicated. 
etary material depends primarily on the energy of the particles and the physical characteristics of the planetary material. The compositions of SW and SEP are very close to solar composition with proton and alpha particle being the dominant species (with a $\alpha / p$ ratio of $\leq 0.1$ ); the abundance of heavier ions $(Z \geq 6)$ accounts for less than a few percent of the total. The range of low energy (KeV/amu) SW ions is less than a thousand angstrom in material of silicate composition and direct implantation is the primary mode of interaction of SW with planetary material exposed on bodies devoid of an atmosphere or a global magnetic field. Rocks and soils exposed unshielded on the lunar surface have copious records of implanted SW ions, and this should be true for samples exposed on asteroidal surfaces as well. In fact, a certain group of meteorites, known as gas-rich meteorite, do contain records of implanted SW ions. Obviously, some of the constituent components of the gas-rich meteorites were exposed unshielded to SW ions on their parent bodies (asteroids) prior to their compaction leading to formation of these meteorites (see, e.g., Goswami et al., 1984). The best tracers of solar wind records in planetary materials are the isotopes of light noble gases (e.g., ${ }^{4} \mathrm{He},{ }^{20} \mathrm{Ne},{ }^{36} \mathrm{Ar}$ ).

The SEP are characterized by relatively high energy (1$100 \mathrm{MeV} / \mathrm{amu}$ ) with an average range of a few millimeters in material of silicate composition. Thus SEP interaction records will be seen in samples exposed on Moon and asteroids with very little or no shielding. The low energy solar particles may get directly implanted, while the relatively higher energy solar proton and alpha particles can initiate nuclear reactions leading to production of both stable and radioactive nuclides; the nuclides produced will depend on the composition of the planetary target material. The range of the high energy GCR protons and alpha particles (typical energy a few GeV/amu) is more than a meter in material of silicate composition and these particles can produce stable and radioactive nuclides in planetary materials up to a shielding depth of couple of meters. A list of stable and radioactive nuclides produced by SEP and GCR interactions with planetary materials are given in Table 1; these nuclides are detected in lunar samples and meteorites.

Even though the abundance of heavier ions in SEP and GCR is much lower, the very heavy $(Z>20)$ ions in SEP and GCR can produce solid state damage in silicate material that can be permanent in nature. Such damage trails are formed near the end of the range of the energetic heavy ions when their ionization loss rate reaches very high values. Such damage trails can be seen directly by transmission electron microscope and may be enlarged by chemical etching and seen using a scanning electron or optical microscope and are termed as nuclear tracks (see, e.g., Fleischer et $a l ., 1975)$. The tracks produced by SEP can be easily distinguished based on the following features: i) the low energy SEP tracks are seen only in the very near surface layers of planetary material exposed to SEP, ii) SEP tracks are characterized by a steep gradient in track density $\left(\# / \mathrm{cm}^{-2}\right)$ over a small range $(\sim 0.01 \mathrm{~cm})$, a reflection of the steeply falling energy spectrum characteristic of SEP (see Fig. 1) and iii) SEP track densities are much higher than the background GCR track densities because of the high flux of low energy SEP heavy ions. Photomicrographs of SEP tracks in lunar
Table 1. Cosmogenic nuclides detected in lunar samples and meteorites*

\begin{tabular}{ccl}
\hline Nuclide & Half-life (yr) & \multicolumn{1}{c}{ Major Targets } \\
\hline${ }^{37} \mathrm{Ar}$ & 0.096 & $\mathrm{Ca}, \mathrm{K}, \mathrm{Ti}, \mathrm{Fe}, \mathrm{Ni}$ \\
${ }^{56} \mathrm{Co}$ & 0.213 & $\mathrm{Fe}, \mathrm{Ni}$ \\
${ }^{54} \mathrm{Mn}$ & 0.855 & $\mathrm{Fe}, \mathrm{Ni}, \mathrm{Mn}$ \\
${ }^{22} \mathrm{Na}$ & 2.60 & $\mathrm{Mg}, \mathrm{Al}, \mathrm{Si}$ \\
${ }^{55} \mathrm{Fe}$ & 2.7 & $\mathrm{Fe}, \mathrm{Ni}$ \\
${ }^{60} \mathrm{Co}$ & 5.26 & $\mathrm{Co}, \mathrm{Ni}$ \\
${ }^{44} \mathrm{Ti}$ & 59.2 & $\mathrm{Ti}, \mathrm{Fe}, \mathrm{Ni}$ \\
${ }^{39} \mathrm{Ar}$ & 269 & $\mathrm{Ca}, \mathrm{Ti}, \mathrm{Fe}, \mathrm{Ni}$ \\
${ }^{14} \mathrm{C}$ & 5730 & $\mathrm{O}$ \\
${ }^{59} \mathrm{Ni}$ & $7.6 \times 10^{4}$ & $\mathrm{Fe}, \mathrm{Ni}$ \\
${ }^{41} \mathrm{Ca}$ & $1.0 \times 10^{5}$ & $\mathrm{Ti}, \mathrm{Ca}, \mathrm{K}$ \\
${ }^{81} \mathrm{Kr}$ & $2.1 \times 10^{5}$ & $\mathrm{Rb}, \mathrm{Sr}, \mathrm{Zr}, \mathrm{Y}$ \\
${ }^{36} \mathrm{Cl}$ & $3.0 \times 10^{5}$ & $\mathrm{Cl}, \mathrm{Ca}, \mathrm{K}$ \\
${ }^{26} \mathrm{Al}$ & $7.1 \times 10^{5}$ & $\mathrm{Mg}, \mathrm{Al}, \mathrm{Si}$ \\
${ }^{10} \mathrm{Be}$ & $1.5 \times 10^{6}$ & $\mathrm{O}$ \\
${ }^{53} \mathrm{Mn}$ & $3.7 \times 10^{6}$ & $\mathrm{Fe}, \mathrm{Ni}$ \\
${ }^{129} \mathrm{I}$ & $1.57 \times 10^{7}$ & $\mathrm{Te}, \mathrm{Ba}, \mathrm{REE}$ \\
${ }^{40} \mathrm{~K}$ & $1.28 \times 10^{9}$ & $\mathrm{Ca}, \mathrm{Ti}, \mathrm{Fe}, \mathrm{Ni}$ \\
$\mathrm{He}$ & stable & $\mathrm{O}, \mathrm{Mg}, \mathrm{Al}, \mathrm{Fe}, \mathrm{Ni}$ \\
$\mathrm{Ne}$ & stable & $\mathrm{Na}, \mathrm{Mg}, \mathrm{Al}, \mathrm{Si}$ \\
$\mathrm{Ar}$ & stable & $\mathrm{Cl}, \mathrm{Ca}, \mathrm{Fe}, \mathrm{Ni}$ \\
$\mathrm{Kr}$ & stable & $\mathrm{Br}, \mathrm{Rb}, \mathrm{Sr}, \mathrm{Y}, \mathrm{Zr}$ \\
$\mathrm{Xe}$ & stable & $\mathrm{Te}, \mathrm{I}, \mathrm{Ba}, \mathrm{REE}$ \\
\hline${ }^{2}$ & &
\end{tabular}

${ }^{*}$ Cosmogenic stable elements/isotopes other than the noble gases are extremely difficult to detect and are not listed.

and asteroidal (meteorite) samples are shown in Fig. 3.

The primary mode of interaction of dust grains with rock and soil grains exposed on the surface of a planetary body devoid of an atmosphere is direct impact that leads to partial or complete destruction of the impacting grain due to localized melting and evaporation. Such impacts also produce microcraters, erosion of surface material by abrasion and at times fracturing and catastrophic destruction of the target rock/soil grains. Impact of dust grains on lunar or asteroidal regolith lead to formation of microcraters on individual regolithic components as well as formation of shock melted glass, glassy spherules and glassy-bonded agglutinates. They also cause microscale erosion of the rock/soil target and induce small scale gardening of the surficial regolith layers. Impacts of relatively large meteoroids (millimeter to centimeter in size) lead to mixing and turnover of the top few centimeter of the regolith while impacts of much larger objects lead to excavation and redistribution of material and controls the growth of regolith on lunar/asteroidal surface. A schematic representation of the various types of interactions of energetic particles and dust grains in the case of asteroidal/lunar regolith is shown in Fig. 4. 

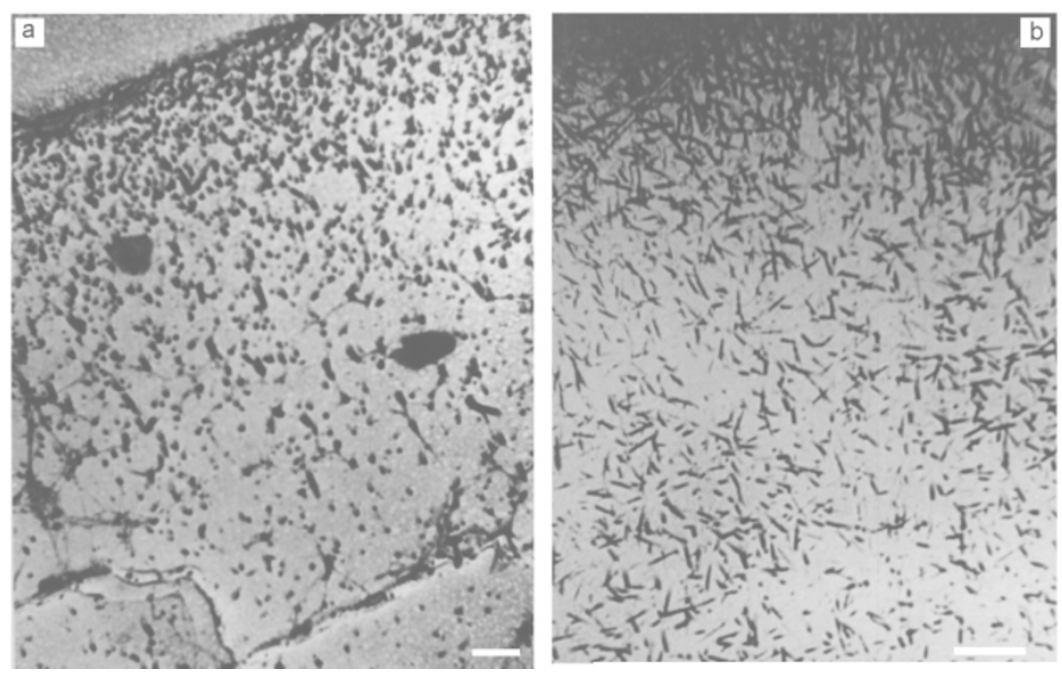

Fig. 3. Photomicrographs of tracks produced by solar flare iron-group heavy ions in a lunar grain (a) and in a grain from the gas-rich meteorite Kapoeta (b) taken by an electron microscope and an optical microscope, respectively. Scale bars are 2 and 10 microns.

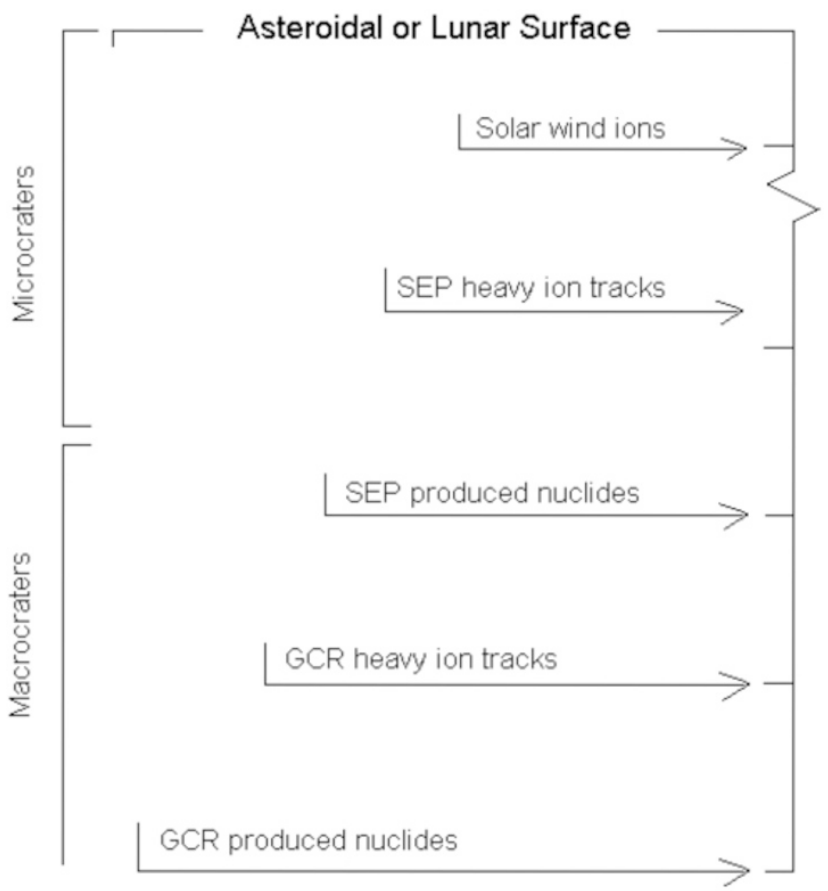

Fig. 4. A schematic representation of approximate depths up to which implantation of solar wind and production of nuclear tracks and stableand radio-nuclides by SEP and GCR can take place on asteroidal or lunar regolith. The depths up to which micro- and macro-craters can induce reworking of the lunar regolith is also indicated.

\section{SW, SEP and Micrometeoroid Records on Lu- nar and Asteroidal Surface}

Extensive studies of interactions of SW, SEP and micrometeoroids with lunar surface material were carried out during the last three decades. While the samples collected from the lunar surface contain records of the current epoch, the samples of drive core and drill core tubes provide information on SW, SEP and micrometeoroid activity at distant epochs going back to a couple of billion years in the past. Some of the lunar regolith breccia contains individual components that were exposed to SW and SEP at even earlier epochs. It is, however, important to note that all these records represent long-term averages integrated over time periods of $10^{3}$ to $10^{6}$ years, the effective surface exposure duration of the analyzed samples. In contrast to the abundant records of low energy SW, SEP and micrometeoroid interactions in lunar samples, similar records in the case of asteroids are limited to those preserved in gas-rich meteorites and they refer to the precompaction era of these meteorites. Unfortunately, it is rather difficult to measure the compaction ages of meteorites. The presently available data suggest that the precompaction records seen in the gas-rich carbonaceous meteorites could be from a very early epoch in the evolution of the solar system dating back to $>4.2 \mathrm{Ga}$ before present (see, e.g., Goswami et al., 1984; Caffee and Macdougall, 1988). In the following, a brief summary of some of our present understanding of the evolution of asteroidal regoliths is presented along with a comparison with the lunar case. Information about characteristics of energetic particles and dust grains in the asteroidal space and about asteroidal surface dynamical processes that we may be able to obtain from analyses of asteroidal surface samples to be returned by the MUSES-C mission is also discussed.

\section{Asteroid Regolith Dynamics}

The evolution of regolith on planetary objects such as the Moon or asteroids depends on the nature and flux of the impacting meteoroids and impact crater dynamics. The growth of regolith in these objects is considered to be due to the widely spread distribution of impact ejecta following large impacting events. Thus, at any given point on the surface of these bodies, regolith grows in discrete steps with deposition of impact ejecta blankets as well as occasional removal of material. The growth process also depends on the size, mass and other physical properties of the planetary objects. For example, the low gravity of the asteroids leads to a much wider distribution of impact ejecta over its surface compared to that on the lunar surface. The physical/compositional properties of the asteroids also affect 
Table 2. Features characteristic of micrometeoroid impacts and SW and SEP irradiations observed in gas-rich meteorites and lunar soils and breccias*.

\begin{tabular}{lccc}
\hline \multirow{2}{*}{ Characteristic features } & \multicolumn{2}{c}{ Gas-rich Meteorites } & \\
\cline { 2 - 3 } & $\begin{array}{c}\text { Chondrites } \\
\text { and Achondrites }\end{array}$ & $\begin{array}{c}\text { Carbonaceous } \\
\text { chondrites }\end{array}$ & Lunar samples \\
\hline Impact glass & rarely $>1 \%$ & - & Up to $50 \%$ \\
Agglutinates & rare & - & Up to $60 \%$ \\
Glassy spherules & rare & - & Up to $10 \%$ \\
Shock features & common & not obvious & common \\
Micrometeoroid impact craters & rare & rare & ubiquitous \\
Helium-4 (cc STP/g) & $10^{4}-10^{5}$ & $10^{3}-10^{4}$ & $>10^{7}$ \\
Grains with SEP tracks $(\%)$ & $1-15$ & $<1-10$ & $10-100$ \\
\hline
\end{tabular}

*For source of data, see, Goswami et al. (1984).

crater dynamics and hence regolith characteristics (Housen et al., 1983; Asphaug et al., 1996). Impact induced seismic disturbance can also play an important role in shaping regoliths on smaller asteroidal bodies. Theoretical modeling of asteroidal regoliths (Housen et al., 1979; Langevin and Maurette, 1980; Housen and Wilkening, 1982; see also articles in this volume) suggest that strong (basaltic) asteroids of a few hundred kilometers in size can develop regoliths that may extend up to a kilometer or more in depth. Obviously, the rate of growth of such regoliths has to be much higher than in the Moon (average regolith thickness $\sim 10$ meters) and this will result in much lower surface exposures of asteroidal regolithic components compared to the lunar case. One would therefore expect the asteroidal regolith to be immature by lunar standard in the sense that the magnitude of various effects caused by interaction of low energy SW and SEP (e.g., implanted SW ions, SEP produced nuclides and nuclear tracks) and by impact of micrometeorites (e.g., microcraters, comminution of grains and fragments, shock melted glass, glassy spherules, agglutinates etc.) will be significantly lower in asteroidal regoliths. The modeling studies also indicate that relatively small $(<10 \mathrm{~km})$ asteroids that are compositionally strong will hardly have any regolith, while compositionally weak (e.g., carbonaceous) small asteroids may have centimeter- to meter-scale regolith (Housen et al., 1979; Michikami, 2001). The possibility that larger asteroids may have experienced cycles of regolith growth, fragmentation and reassembly is also suggested.

Direct information about the nature of asteroidal regoliths comes from studies of gas-rich meteorites belonging to the chondrite, achondrite and the carbonaceous group. We show in Table 2 a comparison of several features charcteristic of near surface exposure of lunar samples and gas-rich meteorites on lunar and asteroidal regoliths, respectively. The features for the non-carbonaceous gas-rich meteorites (low SW content, lower SEP track densities, lack of microcraters, impact glass and agglutinates) clearly suggest that the regoliths on their parent asteroids were extremely immature compared to the lunar regolith. These features are compatible with the theoretically modeled fast growth rate of regoliths in parent bodies of strong meteorites noted earlier. The case for the gas-rich carbonaceous chondrites is more complex and it appears that regolithic activity in the parent bodies of these meteorites was very minimal. As already emphasized, these features of asteroidal regoliths refer to the precompaction era of the gas-rich meteorites (see, e.g., Goswami et al., 1984; Caffee and Macdougall, 1988).

Our knowledge of contemporary regolith on asteroidal surfaces is rather scanty and restricted to remote observation by various flyby missions to several minor objects that include Phobos (Mars Global Surveyer), 243 Ida and 951 Gaspra (Galileo) and 253 Mathilde and 433 Eros (Near Shoemaker). The mean size of these objects ranges from about 12 to $53 \mathrm{~km}$ and of the four asteroids, three belong to $\mathrm{S}$ type and one (Mathilde) to $\mathrm{C}$ type. High resolution imaging of the surfaces of these objects provided detail information on crater morphology, distribution and extent of burial of ejecta blocks and downslope movement of material along crater walls or inclined slopes. Analysis of these features suggest the presence of a few tens to several tens of meters of regolith consisting of loosely consolidated fine material as well as meter-sized blocks on these objects (Carr et al., 1994; Sullivan et al., 1996; Veverka et al., 1997, 2001; Thomas et al., 2000). While the spacecraft observations confirm the presence of regolith in moderate-sized asteroids, the regolith thickness in such objects appears to be much larger than model predictions. A sample return mission like the Muses-C will be extremely important in this context. Analysis of features representative of regolithic activity (see Table 2) in the returned samples will allow us to compare the nature of the contemporary asteroidal and lunar regoliths and test model predictions.

\section{SW and SEP Records in Lunar and Asteroidal Space}

The records of energetic particle (SW, SEP and GCR) interactions with lunar samples have provided a wealth of information about the energetic particle environment in the lunar (1 AU) space during the contemporary as well as distant epochs in the past. Studies of SW implanted noble gases and nitrogen in lunar soils and breccias suggest possible temporal variations in solar wind $\mathrm{He}$ and $\mathrm{N}$ isotopic compositions as well as abundance of Xe over the last 3 billion years. Several proposals have been put forward to explain these obser- 
vations (see, e.g., Kerridge et al., 1991; Geiss and Bochsler, 1991). Differences in SW and SEP isotopic compositions of specific elements have also been observed. The most thoroughly studied example being isotopic composition of $\mathrm{Ne}$; the ${ }^{20} \mathrm{Ne} /{ }^{22} \mathrm{Ne}$ abundance ratio in SEP is found to be about $25 \%$ lower than in SW (see, e.g., Wieler, 1998). Studies of activity of SEP produced radionuclides of different halflives in lunar samples have also provided information on the flux of solar protons averaged over the mean-lives of the radionuclides. The presently available data suggest that the solar proton flux averaged over the last few million years is less than the value averaged over shorter time scales of $\sim 10^{4}$ to $10^{5}$ years (see, e.g., Reedy, 1998). Studies of nuclear track records in lunar samples indicate that the spectral shape of the solar flare heavy ion energy spectra remained nearly the same over the last few billions years although variation in solar flare intensity during different epochs in the past cannot be ruled out. Further, these studies also led to the first observation of preferential enhancement of very heavy nuclei ( $Z \geq 30$ ) over Fe group nuclei in solar flares; such enhancement has also been seen later in contemporary solar flares (see, e.g., Goswami, 1991).

Interaction records of SW and SEP present in gas-rich meteorites provide information on the characteristics of SW and SEP during the precompaction era of these meteorites. Noble gas studies of bulk samples of gas-rich meteorites reveal excess abundance of light noble gases ( $\mathrm{He}, \mathrm{Ne}, \mathrm{Ar}$ ) and the isotopic composition of $\mathrm{Ne}$ in these meteorites clearly indicates the presence of a solar (SW) component in addition to the trapped and GCR components. The difference in SW and SEP neon isotopic composition inferred from lunar sample studies is also seen in data obtained for bulk samples (mineral separates) of gas-rich meteorites. However, quantitative studies of interaction records of SW and SEP in gasrich meteorites are rather difficult as only a small fraction (typically a few percent) of the individual mineral grains of these meteorites contain records of precompaction SW and SEP interactions (Table 2; see also Goswami et al., 1984). Identification of individual grains with solar flare tracks in gas-rich meteorite samples provides a way for attempting a quantitative approach. High track densities compared to the background GCR track densities and a gradient in track density (see Fig. 3) are the two criteria used for identifying individual grains exposed to SEP. Studies of solar flare track records in such grains from different gas-rich meteorites suggest a similarity in the shape of the Fe-group nuclei energy spectra during the precompaction era of these meteorites with those seen in lunar samples exposed to SEP during more recent epochs. This similarity also extends to SEP track record for the contemporary era as recorded in the Surveyer Camera glass that was retrieved from the Moon after an exposure for a 2.6 year period during 1967 to 1969 (Fig. 5; see also Goswami, 1991). The similarity in the records from such widely separated epochs suggest that the electromagnetic processes involved in flare acceleration and propagation have not changed significantly over the last four billion years.

Individual grains from gas-rich meteorites with solar flare track records have also been analyzed to look for solar proton produced stable nuclides using laser ablation mass spec-

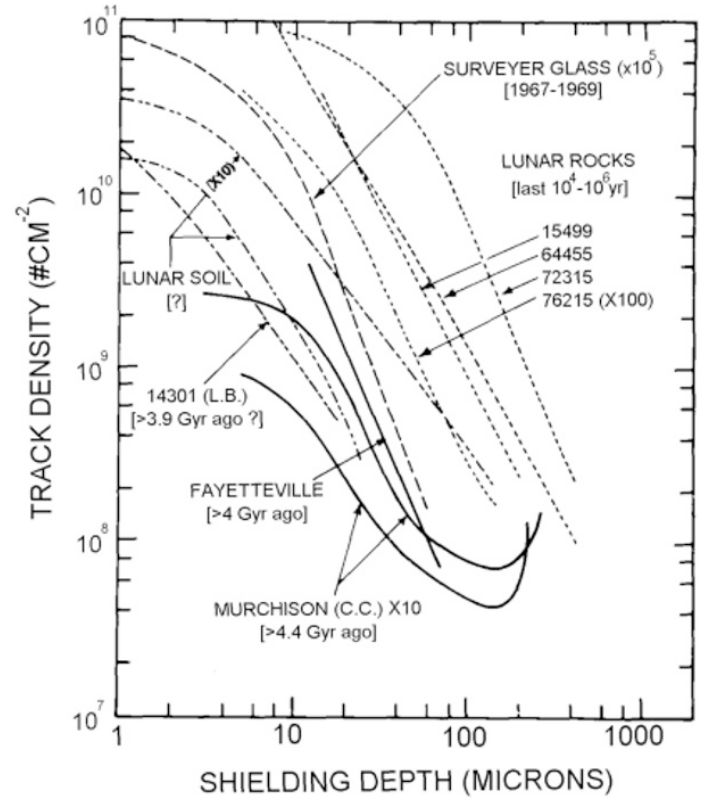

Fig. 5. Solar flare heavy ion track density profiles in lunar and asteroidal (gas-rich meteorite) samples. The approximate epoch of solar flare irradiation of the different samples is also indicated. Contemporary data, based on studies of Surveyer camera glass, is also shown (L.B. = lunar breccia; C.C. carbonaceous chondrite; see Goswami, 1991 for source of data).

trometry or in vacuo step-etching and on-line mass spectrometry (see, e.g., Hohenberg et al., 1990; Wieler et al., 1986). Results obtained from a combined study of nuclear track and noble gas records in individual grains from several gas-rich meteorites belonging to the carbonaceous chondrite group provided the first experimental evidence to suggest that the Sun has gone through an active early (T-Tauri) phase (Hohenberg et al., 1990; Caffee et al., 1991). Theoretical modeling of early evolution of solar type stars as well as astronomical observations of young Sun-like (T-Tauri) stars do suggest an active early phase for the Sun (see, e.g., Feigelson et al., 1991). However, unlike the lunar case, we do not have information about the composition and intensity of SW, SEP and GCR particles during the present epoch for the region of interplanetary space span by the asteroids. Studies of samples to be returned by the Muses- $\mathrm{C}$ mission will be extremely useful in this regard. Specific questions such as possible temporal variation in the SW isotopic composition in the asteroidal space, similar to those seen at $1 \mathrm{AU}$ (lunar) space, can also be addressed by comparing the data for the returned samples with those for the gas-rich meteorites. Studies of activity of SEP produced radionuclides of different half-lives will also allow us to check if the SEP flux averaged over the last few $10^{4}$ to $10^{5}$ years is indeed higher than the long-term ( $\geq \mathrm{Ma}$ ) averaged value, as indicated by the lunar sample data. Most of the important diagnostic radionuclides (e.g., ${ }^{14} \mathrm{C},{ }^{41} \mathrm{Ca},{ }^{36} \mathrm{Cl},{ }^{26} \mathrm{Al}$, and ${ }^{10} \mathrm{Be}$ ) can be analyzed in small samples using accelerator mass spectrometric techniques. 


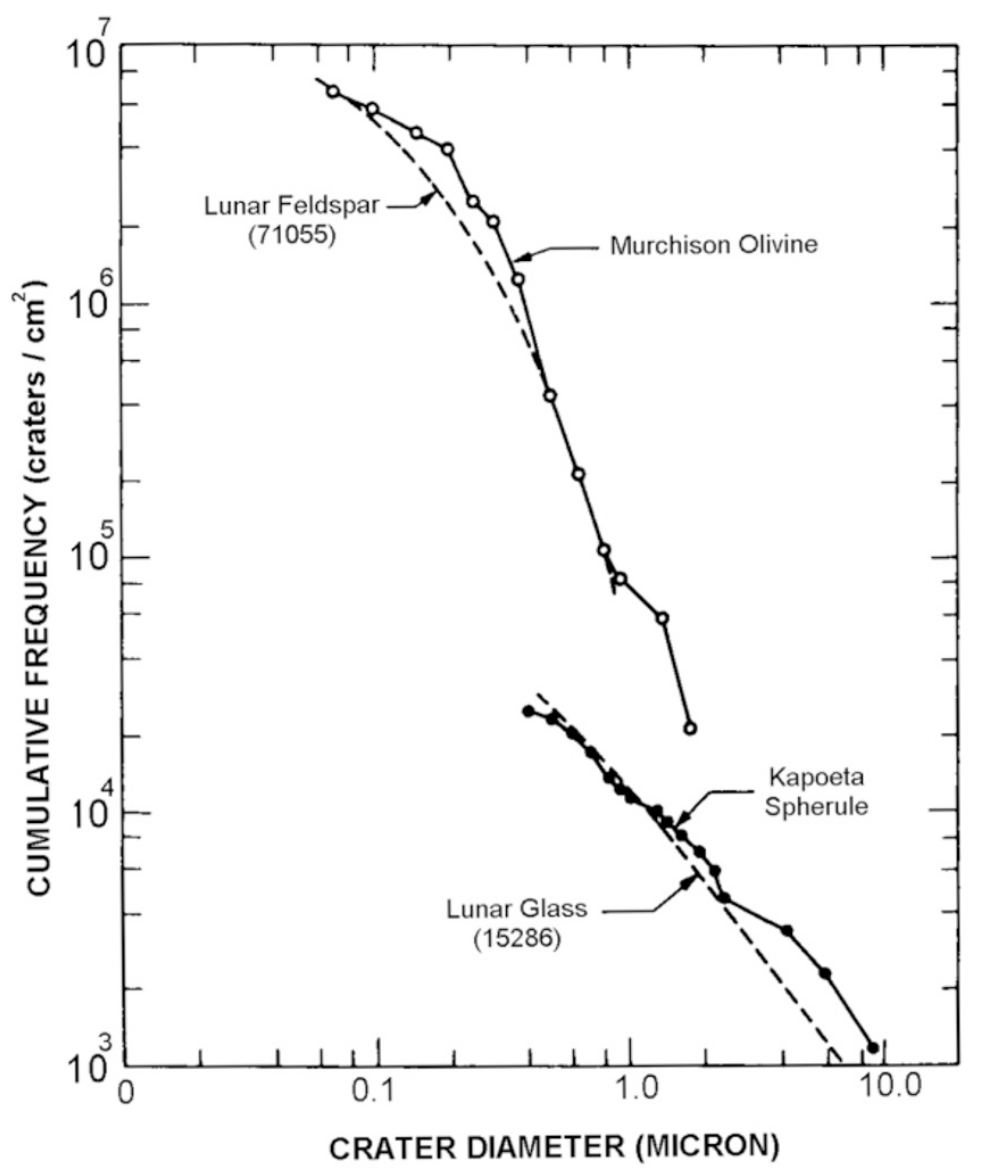

Fig. 6. A comparison of frequency distribution of microcraters on samples from Moon and gas-rich meteorites Murchison and Kapoeta. The lunar data are arbitrarily normalized at 0.5 micron for comparison with the meteorite data.
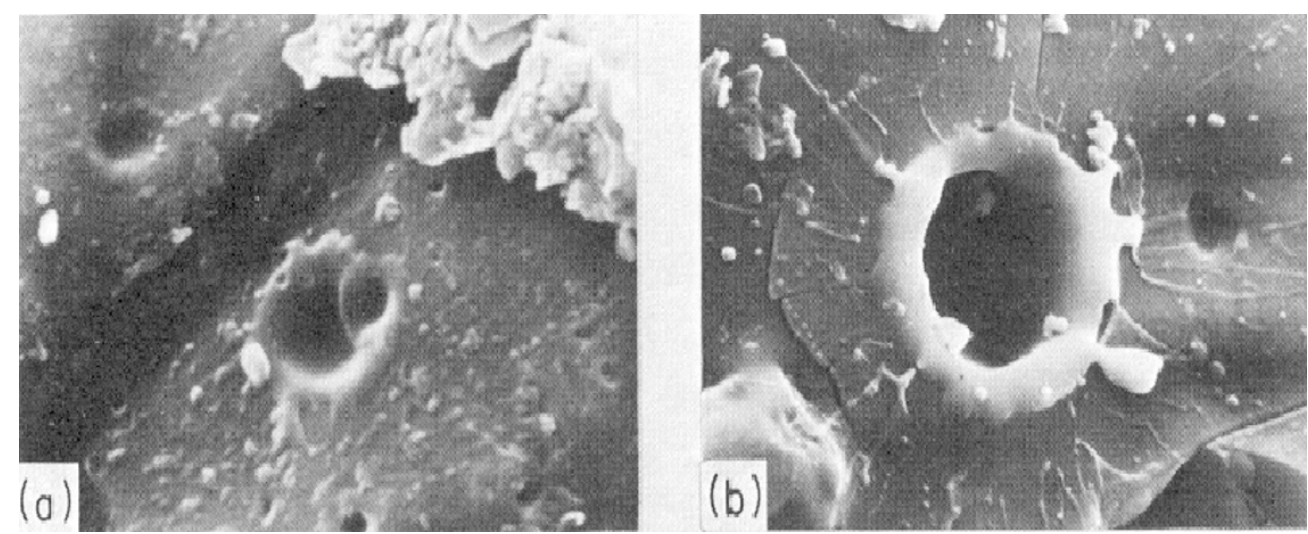

Fig. 7. Impact microcrater on the surface of an olivine grain from the carbonaceous chondrite, Murchison (a) and on the surface of a lunar grain (b). Crater diameters are 0.85 and 2.2 microns, respectively.

\section{Energetic Dust Grains in Lunar and Asteroidal Space}

Extensive studies of micrometeoroid impact induced features, such as, microcraters, impact glass, glassy spherules and glassy-bonded agglutinates in lunar samples have provided us very useful information on the flux of interplanetary dust grains at $1 \mathrm{AU}$ space and also their physical characteristics, such as density and velocity. Similar information is meager in the case of asteroidal samples due to the paucity of micrometeoroid impact records in gas-rich meteorites. Further, identification of such records in gas-rich meteorites is very difficult. It is necessary to isolate individual grains in these meteorites that were exposed on asteroidal surface (using solar flare tracks as tracer) and then look for possible presence of impact induced features on the surfaces of these grains. Brownlee and Rajan (1973) first reported observation of microcraters on the surface of a chondrule-like object isolated from the gas-rich howardite, 
Kapoeta. This was followed up by a detail study of the gasrich carbonaceous chondrites (Goswami et al., 1976). Several salient features of the dust grain population in the asteroidal belt that can be inferred from the available data are: i) the size-frequency distribution of the dust grains in the asteroidal space is similar to that in the lunar (1 AU) space (Fig. 6), ii) the lower depth to diameter ratio of microcraters on meteoritic grains compared to those on lunar grains (see Fig. 7) suggest that the impact velocity of dust grains on asteroidal surface is much lower $\left(\sim 5 \mathrm{~km} \mathrm{~s}^{-1}\right)$ compared to the typical impact velocity of $\sim 20 \mathrm{~km} \mathrm{~s}^{-1}$ on the lunar surface, and iii) the dust grain flux in asteroidal space at $>4.2$ Ga before present could have been about twenty times higher than the contemporary flux at 1 AU. Unfortunately, our knowledge of contemporary flux of dust grains in the asteroidal space is limited to the data obtained by dust detectors on board interplanetary spacecrafts such as Pioneer 10 and 11 as they traversed the asteroidal zone (see, e.g., Dohnanyi, 1976; McDonnell, 1978). The Muses-C mission assume significance in this context as the samples returned by this mission will hopefully provide new information on the dust grain population in the asteroidal space for the contemporary epoch.

\section{The Muses-C Mission and New Possibilities}

The Muses-C mission that will bring back surficial samples from a small size asteroid will be extremely important for advancing our knowledge of the energetic particles and dust grains in the asteroidal region during the present epoch. Comparative studies of lunar and asteroidal surface samples will also improve our understanding of the surface dynamical processes operating in these two distinctly different types of planetary objects. Studies of SW, SEP, GCR and dust grain impact records in the returned asteroidal surface samples and in gas-rich meteorites will provide information on possible temporal variations in the characteristics of energetic particles and dust grains in asteroidal space.

The sample collection technique to be utilized in the Muses-C mission is expected to mix up material from the top layer of the asteroidal regolith and the retrieved sample will be analogous to the scoop samples of lunar regolith collected during the Apollo missions. It will be important to identify individual components that were residing on the very surface of the asteroidal regolith as well as those with varying degree of shielding at the time of sampling. Nondestructive approach such as studies of microcraters and nuclear tracks produced by SEP and GCR particles will be ideal to infer shielding conditions of the returned samples. The samples thus labeled can be analyzed for SW and SEP and GCR produced stable- and radio-nuclides, using suitable techniques developed to handle microscopic samples. The resultant data set will allow us to infer the composition and intensity of SW and SEP in the asteroidal region during the present epoch and also the details of asteroid surface dynamical processes. The target asteroid of Muses-C mission [(25413)1998SF36] is smaller than a $\mathrm{km}$ in size and although very little is known about its composition, there is a distinct possibility that it may have a sub-meter to metersized regolith. Theoretical modeling of regolith development on small asteroids suggests that compositionally weak kilometer-sized asteroids may develop regolith that could extend up to a meter in depth. The recent spacecraft observations also suggest that regolith thickness even in compositionally stronger small asteroid could be higher than model prediction. We may therefore look forward to obtain a wealth of information on contemporary energetic particle and dust grain environment in the asteroidal zone from the samples to be returned by the Muses-C mission.

\section{References}

Ashworth, D. G., Lunar and planetary impact erosion, in Cosmic Dust, edited by J. A. M. McDonnell, pp. 427-526, Wiley-Interscience Publication, 1978.

Asphaug, E., J. M. Moore, D. Morrison, W. Benz, M. C. Nolan, and R. Sullivan, Mechanical and geological effects of impact cratering on Ida, Icarus, 120, 158-184, 1996.

Biswas, S., N. Durgaprasad, B. Mitra, and A. Dutta, Anuradha and low energy cosmic rays, Space Sci. Rev., 62, 3-65, 1993.

Brownlee, D. E., Cosmic dust: Collection and research, Ann. Rev. Earth Planet. Sci., 13, 147-173, 1985.

Brownlee, D. E. and R. S. Rajan, Micrometeorite craters discovered on chondrule like objects from Kapoeta meteorite, Science, 182, 13411344, 1973.

Caffee, M. W. and J. D. Macdougall, Compaction ages, in Metorites and the Early Solar System, edited by J. F. Kerridge and M. S. Matthews, pp. 289-298, Arizona Univ. Press, Tucson, 1988

Caffee, M. W., J. N. Goswami, C. M. Hohenberg, K. Marti, and R. C. Reedy, Irradiation records in meteorites, in Metorites and the Early Solar System, edited by J. F. Kerridge and M. S. Matthews, pp. 205-245, Arizona Univ. Press, Tucson, 1988.

Caffee, M. W., C. M. Hohenberg, R. H. Nichols, Jr., C. T. Olinger, R. Wieler, A. Pedroni, P. Signer, T. D. Swindle, and J. N. Goswami, Do meteorites contain irradiation records from exposure to an enhancedactivity Sun?, in The Sun in Time, edited by C. P. Sonett, M. S Giampapa, and M. S. Matthews, pp. 413-425, Arizona Univ. Press, Tucson, 1991

Carr, M. H., R. L. Kirk, A. McEwen, J. Veverka, P. Thomas, J. W. Head, and S. Murchie, The geology of Gaspra, Icarus, 107, 61-71, 1994.

Dohnanyi, J. S., Sources of interplanetary dust: asteroids, in Lecture Notes in Physics, 48, edited by H. Elsasser and H. Fechtig, p. 143, SpringerVerlag, 1976.

Feigelson, E. D., M. S. Giampapa, and F. J. Vrba, Magnetic activity in pre-main-sequence stars, in The Sun in Time, edited by C. P. Sonett, M. S. Giampapa, and M. S. Matthews, pp. 658-681, Arizona Univ. Press, Tucson, 1991

Fleischer, R. L., P. B. Price, and R. M. Walker, Nuclear Tracks in Solids, 605 pp., Univ. of California Press, Berkeley, 1975.

Gault, D. E., F., Horz, and J. B. Hartung, Effects of microcratering on the lunar surface, Proc. 3rd Lunar Sci. Conf., 3, 2713, 1972.

Gault, D. E., F. Horz, D. E. Brownlee, and J. B. Hartung, Mixing of the lunar regolith, Proc. 5th Lunar Sci. Conf., 2365, 1974.

Geiss, J. and P. Bochsler, Long-time variations in solar wind properties: Possible causes versus observations, in The Sun in Time, edited by C. P. Sonett, M. S. Giampapa, and M. S. Matthews, pp. 98-117, Arizona Univ. Press, Tucson, 1991.

Goswami, J. N., Solar flare heavy ion tracks in extraterrestrial objects, in The Sun in Time, edited by C. P. Sonett, M. S. Giampapa, and M. S. Matthews, pp. 426-444, Arizona Univ. Press, Tucson, 1991.

Goswami, J. N., I. D. Hutcheon, and J. D. Macdougall, Microcraters and solar flare tracks in crystals from carbonaceous chondrites and lunar breccias, Proc. Lunar Sci. Conf. 7th, 543-562, 1976.

Goswami, J. N., D. Lal, and L. L. Wilkening, Gas-rich meteorites: Probes for particle environment and dynamical processes in the inner solar system, Space Sci. Rev., 37, 111-159, 1984.

Grun, E., H. A. Zook, H. Fechtig, and R. H. Giese, Collisional balance of the meteoritic complex, Icarus, 62, 244-272, 1985

Hohenberg, C. M., R. H. Nichols, Jr., C. T. Olinger, and J. N. Goswami, Cosmogenic neon form individual grains of CM meteorites: Extremely long pre-compaction exposure histories or an enhanced early particle flux, Geochim. Cosmochim Acta, 54, 2133-2140, 1990.

Housen, K. R. and L. L. Wilkening, Regoliths on small bodies in the solar system, Ann. Rev. Earth Planet. Sci., 10, 355-376, 1982.

Housen, K. R., L. L. Wilkening, C. R. Chapman, and R. Greenberg, Aster- 
oidal regoliths, Icarus, 39, 317-351, 1979.

Housen, K. R., R. M. Schmidt, and K. A. Holsapple, Crater ejecta scaling laws: Fundamental forms based on dimensional analysis, J. Geophys. Res., 88, 2485-2499, 1983.

Hughes, D. W., Meteors, in Cosmic Dust, edited by J. A. M. McDonnell, pp. 123-185, Wiley-Interscience Publication, 1978.

Kerridge, J. F., P. Signer, R. Wieler, R. H. Becker, and R. O. Pepin, Longterm changes in composition of solar particles implanted in extraterrestrial materials, in The Sun in Time, edited by C. P. Sonett, M. S. Giampapa, and M. S. Matthews, pp. 389-412, Arizona Univ. Press, Tucson, 1991.

Klecker, B., R. A. Mewaldt et al., Anomalous cosmic rays, Space Sci. Rev., 83, 259-308, 1998.

Lal, D., Hard rock cosmic ray archaeology, Space Sci. Rev., 14, 3-102, 1972.

Langevin, Y. and M. Maurette, A model for small body regolith evolution: The critical parameters, Lunar Planet. Sci., XI, 602-604, 1980.

McDonnell, J. A. M., Microparticle studies by space instrumentation, in Cosmic Dust, edited by J. A. M. McDonnell, pp. 337-426, WileyInterscience Publication, 1978.

Michikami, T., Evolution of asteroid regolith layers by cratering, Ph.D. thesis, University of Tokyo, Japan, 2001.

Morrison, D. A. and E. Zinner, Distribution and flux of micrometeoroids, Phil. Trans. Roy. Soc. A, 285, 379, 1976.

Morrison, D. A. and E. Zinner, 12054 and 76215: New measurements of interplanetary dust and solar flare fluxes, Proc. Lunar Sci. Conf. 8th, 841-863, 1977.

Reedy, R. C., Studies of modern and ancient solar energetic particles, Proc. Indian Acad. Sci. (Earth Planet. Sci.), 107, 433-440, 1998.

Reedy, R. C., J. R. Arnold, and D. Lal, Cosmic ray records in solar system matter, Science, 219, 127-135, 1983.

Sullivan, R., R. Greeley, R. Pappalardo, E. Asphaug, J. M. Moore, D.
Morrison, M. J. S. Belton, M. Carr, C. R. Chapman, P. Geissler, R. Greenberg, J. Granahan, J. W. Head, III, R. Kirk, A. McEwen, P. Lee, P. C. Thomas, and J. Veverka, Geology of 243 Ida, Icarus, 120, 119-139, 1996.

Thomas, P. C., J. Veverka, R. Sullivan, D. P. Simonelli, M. C. Malin, M. Caplinger, W. K. Hartmann, and P. B. James, Phobos: Regolith and ejecta blocks investigated with Mars Orbiter Camera images, J. Geophys. Res., 105, 15091-15106, 2000.

Veverka, J., P. Thomas, A. Harch, B. Clark, J. F. Bell, III, B. Carcich, J. Joseph, C. Chapman, W. Merline, M. Robinson, M. Malin, L. A. McFadden, S. Murchie, S. E. Hawkins, III, R. Farquhar, N. Izenberg, and A. Cheng, NEAR's flyby of 253 Mathilde: Images of a C asteroid, Science, 278, 2109-2114, 1997.

Veverka, J., P. C. Thomas, M. Robinson, S. Murchie, C. Chapman, M. Bell, A. Harch, W. J. Merline, J. F. Bell, III, B. Bussey, B. Carcich, A. Cheng, B. Clark, D. Domingue, D. Dunham, R. Farquhar, M. J. Gaffey, E. Hawkins, N. Izenberg, J. Joseph, R. Kirk, H. Li, P. Lucey, M. Malin, L. McFadden, J. K. Miller, W. M. Owen, Jr., C. Peterson, L. Prockter, J. Warren, D. Wellnitz, B. G. Williams, and D. K. Yeomans, Imaging of small-scale features on 433 Eros from NEAR: Evidence for a complex regolith, Science, 292, 484-488, 2001.

Weinberg, J. L. and J. G. Sparrow, Zodiacal light as an indicator of interplanetary dust, in Cosmic Dust, edited by J. A. M. McDonnell, pp. 75122, Wiley-Interscience Publication, 1978.

Wieler, R., The solar noble gas record in lunar samples and meteorites, Space Sci. Rev., 85, 303-314, 1998.

Wieler, R., H. Baur, and P. Signer, Noble gases from solar energetic particles revealed by closed system stepwise etching of lunar soil minerals, Geochim. Cosmochim. Acta, 50, 1997-2017, 1986.

J. N. Goswami (e-mail: goswami@prl.ernet.in) 